\title{
Ethical principles and a practical approach to support policy making through the next phases of the COVID-19 pandemic and beyond
}

\author{
Authors: Zoë Fritz,, Julian L Huppert, ${ }^{\mathrm{B}}$ Kathleen Liddell, ${ }^{\mathrm{C}}$ Richard Holton ${ }^{\mathrm{D}}$ and Jonathan P Fuld ${ }^{\mathrm{E}}$
}

There is an urgent need for an ethical framework to help us address the local and national challenges that we face as clinicians during the COVID-19 pandemic. We propose four key commitments from which a practical and consistent ethical approach can be derived. These commitments are to articulate the needs, rights and interests of the different stakeholders affected by any policy; to be accountable and transparent, recognising that people are autonomous individuals with values and concerns of their own; to consider the impact of our actions on the sustainability of the NHS, infrastructure, service demands and staff welfare; and to treat everybody equitably, with all deserving of consideration and care. Implementing these commitments will require a number of specific actions. We must put in place frameworks enabling clear advocacy for each competing objective; communicate policy and practice effectively to the public; promote integration of decision-making among social, primary, secondary and tertiary care and reduce or stop unnecessary or inefficient interventions; minimise health inequalities; and build spare capacity into the system.

In this article, we expand on these actions, and note the legal context in which this would be delivered.

KEYWORDS: Ethics, policy, COVID-19, law

DOI: $10.7861 /$ clinmed.2020-0843

\section{Introduction}

The 3 months from March to May 2020 saw what we had hoped would be the highest peak of the COVID-19 pandemic in the UK.

Authors: ${ }^{\mathrm{A}}$ Consultant in acute medicine, The Healthcare Improvement Studies (THIS) Institute, Cambridge, UK and Cambridge University

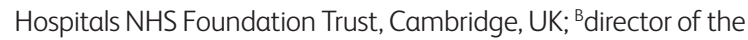
Intellectual Forum, Jesus College, Cambridge; visiting professor in the Policy Institute, King's College London; 'director of Centre of Law, Medicine and Life Sciences and reader in intellectual property and medical law, University of Cambridge, Cambridge, UK; Pprofessor of philosophy, University of Cambridge, Cambridge, UK; ${ }^{E}$ consultant in acute and respiratory medicine, Cambridge University Hospitals NHS Foundation Trust, Cambridge, UK
Many papers have already been written about this extraordinary period: the logistical, clinical and emotional challenges we have faced; the inequities which have been unmasked; the difficulty of prioritising among patients and among different care settings; the lack of integrated care; the focus on ventilator allocation and construction at the expense of care homes; the presence - and lack - of ethical guidance. ${ }^{1}$ As cases rise again we need to think about how to create a framework which will provide the structure to address future challenges.

There is also an opportunity to improve ways of working beyond the needs of this pandemic. The last few months have seen rapid changes in clinical practice, with institutions and individuals trying new approaches to the delivery of care that would in normal times have involved multiple bureaucratic steps. Although these are for the most part operational changes, they all have ethical aspects which need to be considered. And while, in some respects, people are keen to return to work as before, we must not lose the positive changes that have come from this time of unrest: the shared goals that have led to better collaborations and to more integrated thinking.

A pandemic shifts emphasis within clinicians' ethical and professional frameworks. For example, the General Medical Council's Duties of a doctor states: 'Make the care of your patient your first concern.'2 In a pandemic, population-level concerns are more explicitly articulated, alongside greater awareness of the importance of looking after staff wellbeing. ${ }^{3,4}$ In truth, we should always be thinking about the patient, other patients and staff, and public health. The pandemic has simply required us to think more carefully about balancing and prioritising the legal and ethical interests of all these groups, and about extending them to future groups.

So what ethical framework should we use, and how should it be applied? Working together in our System-wide Ethical Committee and extrapolating on work done by Fritz and Cox, ${ }^{5}$ who in turn draw on Rawls and Scanlon, we propose four principles from which a practical and consistent approach can be derived.

First, we must make a commitment to articulating the needs, rights and interests of the different stakeholders affected by any policy. In this pandemic, the needs of those infected with COVID-19 conflicted with the needs of those suffering with nonCOVID-19-related illnesses and long-term conditions; we needed to make and enact policies which achieved the best possible health outcomes for both groups. Alongside these groups, we 
needed to protect the workforce looking after all of these patients and to sustain a health service which would be able to look after future generations of patients. As we move on, different groups will be identified whose perspectives must also be articulated.

Second, we must make a commitment to be accountable and transparent, recognising that people are autonomous individuals with values and concerns of their own. This means that people - at individual and population levels - should be kept as informed as possible; they should have the chance to express their views on matters that affect them; their views about their treatment and care should be appropriately respected; and ultimately decisions taken that affect them should be reasoned and open.

Third, we must make a commitment to consider the impact of our actions on future generations: we must make decisions which are sustainable, ensuring adequate training and support of our staff, and national investment in infrastructure.

Last but by no means least, we must make a commitment to treat everybody equitably, with all deserving of consideration and care. This does not mean giving everyone equal treatment; it means that decisions to treat people differently must be justified on ethically and legally defensible grounds (see Box 1 for more on the legal context).

\section{Recommended actions}

Implementing these commitments will require a number of specific actions, which we outline below.

\section{Put in place frameworks enabling clear advocacy for each competing objective}

These need to be established at local, regional, and national levels. Where decisions are being made, conflicting demands (eg among different patient or staffing groups, or across care settings) should be identified and considered. This is not the same as ensuring there is representation from each group: it means documenting all of the different groups affected, and assigning individuals as 'leads' for each group. We do this for patients who lack capacity in appointing an independent mental capacity advocate (IMCA), and we should do this to ensure that those groups with less power, and less strong voices, are heard in discussions about policy. It also helps to prevent inappropriate weight being given to the objective that is most prominent at any given time. This will be important as we make ongoing decisions about distribution of personal protective

\section{Box 1. The legal context}

During the first peak of the pandemic, aspects of medical law other than the familiar topics of medical negligence, euthanasia and confidentiality came to the fore. Healthcare providers, doctors, public health officers, care home managers and many more were forced rapidly to consider how the law governs people and resources during an extreme public health challenge that poses risks to all, but to some more than others. ${ }^{6,7}$

It became clear we have a legal system that accommodates the social impulse, in an emergency, to use available resources to save the most lives. But fortunately for the weak, vulnerable, and minorities, the law imposes checks, balances and limits, so utilitarian reasoning cannot override all other concerns. Although far from perfect, our legal system, under the Human Rights Act of 1998, protects cornerstone fundamental human rights, including an individual's right to life, their autonomy, their movement and their equality, while simultaneously striving to give appropriate weight to other more public interests, such as priority-setting, monitoring and surveillance, and compulsory detention to protect other people from infectious disease.

A significant example during the first wave of the pandemic was the rapid development of policies for allocation of ventilators, dialysis machines, and intensive care unit (ICU) beds. The law largely, but not entirely, leaves decisions about the distribution of scarce healthcare resources to policy makers and administrative agencies, respecting the political judgments that must be made. However, the State cannot cause degrading treatment, and it must protect patients from intentional deprivation of life. The extent to which doctors can manipulate a patient's body without their consent is also legally limited. These legal conditions, enforceable under the Human Rights Act 1998, judicial review and potentially the law of negligence and criminal law, meant that the NHS could decide which of two patients should be allocated a ventilator, provided their decision was reasoned and transparent, but could not remove a ventilator from a patient simply because they were only slowly recovering and a stronger patient needed it. These important conditions serve to protect people. The Equality Act 2010 further ensures that allocation decisions do not disadvantage people because of a protected characteristic, such as a disability (eg diabetes, cancer or lung disease). It also requires evidence that a COVID-19 policy is proportionate means of achieving a legitimate aim if it has the indirect effect of disadvantaging people with disabilities, the elderly or people of certain races, and that reasonable adjustments are made so that people with disabilities are not placed at a substantial disadvantage. ${ }^{8}$

Outside acute care, the pandemic has had a massive impact on care homes, where residents often lack decision-making capacity. Fortunately, the Mental Capacity Act 2005 provides a well-established framework in these situations to ensure decision-making is based on residents' best interests rather than expediency. Procedures involving external review must also be followed if actions to isolate a resident mean they are deprived of their liberty (eg locked doors). The area of law known as administrative law, or judicial review, further holds public bodies to account. curbing misuse of powers. Judges can be asked to quash decisions which lack reasons or due process. For example, after a 'letter before action' from solicitors for the Cystic Fibrosis Society, the National Institute of Health and Care Excellence (NICE) adjusted a policy that initially recommended a frailty score tool for prioritisation of ICU resources without pointing out that the scoring system had not been validated for non-elderly patients such as younger people with stable long-term disabilities (for example, cerebral palsy), learning disabilities or autism. ${ }^{9}$

These and other features of the law support the commitments and actions recommended in this article, while not making them specifically mandatory. A conscientious desire to learn and act ethically is still the most important driver of change. 


\section{Box 2. The deliberative citizen process}

If decisions should take account of public opinions, as we suggest in our second commitment, a mechanism must be used to find out what these are. One approach is to ask members of the public directly. This can involve explicit polling questions, such as a recent YouGov poll that revealed that the public would give priority access to ventilators to doctors and nurses, parents, and the armed forces above supermarket workers, members of parliament or the Royal Family. ${ }^{10}$ More complex and subtle questions can also be asked, such as the MIT moral machine experiments, which asked people to consider and vote on which of two groups of people should be killed by an autonomous car if it crashed. ${ }^{11}$ This revealed that there are significantly different opinions around the world, and some opinions that may be seen to be ethically surprising: for example it found that people are more willing to kill a criminal than a dog.

Polling relies on the public having appropriate information to make useful judgements, and does not provide space for discussion and reflection. For that reason, many groups have used more expansive deliberative processes. ${ }^{12}$ These bring a number of people together to discuss issues, with information given from both neutral and partisan sources. They vary in scale from the large-scale Deliberative Opinion Poll, pioneered by Jim Fishkin, ${ }^{13}$ which brings together 500 or so people for a few days, to Citizen's Juries, which assemble 1,224 people, often for half a day. The bigger the group, the more representative, but the greater the cost.

Ireland has used Citizen's Assemblies to drive major changes, including constitutional reforms and the repeal on the ban on abortions. The breath of engagement and discussion were very effective at changing broader public and political opinion. Manchester has run smaller Citizen's Juries to consider questions such as 'To what extent should patients control access to patient records?' and the use of artificial intelligence in healthcare, especially the trade-off between explainability and accuracy. Decisions about what medical services to provide have often been driven by expert clinician preferences, which may not accord with the public and patient experience. More recently, there has been more engagement with patient representatives, which has advantages in understanding lived experience, but does not necessarily reflect the wider views. When difficult decisions have to be made about which services to provide or not to provide, deliberative citizen engagement is a powerful and underused tool to help make choices.

equipment (PPE), the prioritisation and order of reopening services, and all the other pressing decisions to come (see Box 2 for more on the deliberative citizen process).

\section{Communicate policy and practice effectively to the public}

We should produce lay summaries and updates of policy and practice across local, regional and national health care groups. Well-placed trust from the public needs to be earned; to do this, there should be access to deliberations, and publication of key policy decisions and key information. This may provoke some anxiety in the short term but will reduce the spread of disinformation and strengthen trust, and may improve health outcomes. ${ }^{14}$ Accessible information is needed about the reasons for shielding (or stopping shielding), the validity of tests, the level of nosocomial infection and the likely waiting times for resumption of normal services. This will respect individuals' autonomy and allow them to make informed decisions about their own behaviour and how to look after each other and to know what to expect when coming to hospital. In addition to being transparent about processes, we need to be transparent about outcomes (and make sure we choose measurable outcomes with direct relevance to patient care and experience). These include those measures already established in healthcare that have been upended by the cessation of almost all routine care (such time-to-treatment and quality of life measures in chronic disease). In addition, COVID-19 brings specific risks to patients and healthcare environments; new quality indices such as mortality from ICU admission and hospital acquired infections may be needed. These agreed measures need to adopted and implemented at scale so that organisations struggling to support their communities can be helped to provide better outcomes.

Promote integration of decision-making among social, primary, secondary and tertiary care and reduce or stop unnecessary or inefficient interventions

This is essential to ensure inequalities are addressed and inefficiencies diminished. We need to learn from the reactive overemphasis placed on the availability of ventilators at the beginning of the pandemic, while avoidable deaths happened due to inadequate planning in care homes, with failures including insufficient PPE and inappropriate discharges to make hospital beds available. It is possible that this occurred because the voices in the acute trusts were louder, or more visible; we need to ensure all aspects of care provision are balanced. As we restart services, we should also be honest with ourselves - and the public - about which ones are needed. Many services that have historically been provided by the NHS are not shown by evidence to be of sufficient benefit, ${ }^{15}$ 'grommets' being a classical example. ${ }^{16}$ As an integrated community we can do this better. We should not simply aim to return to providing treatments or performing investigations just because we did before, but should use this 'reset' opportunity to ensure that we do the activities that are most worth doing. There can be no better time to seize this opportunity; swathes of services have stopped or adapted, few people use NHS time unnecessarily and all care environments are pressured. NHS leaders can support systems to capitalise on this extraordinary situation to better match our health provision to the needs of the populations they serve.

\section{Minimise health inequalities}

As services are restarted it is imperative that the opportunity is taken to reduce existing health inequalities, rather than allowing them to worsen. Drawing on Rawls' difference principle, ${ }^{17,18}$ services should be preferentially restarted in more disadvantaged areas, or where the beneficiaries are less advantaged. Consideration should also be given to equalities in staff deployment, to ensure the extra load does not land on the worst paid.

\section{Build spare capacity into the system}

To maintain a sustainable health service - and one which can respond flexibly to change in demands - we need to build 'spare 
capacity' into the system. The NHS, in response to financial pressures, has been operating on a 'lean' model with no surplus material, and a supply chain - of staff, beds and equipment which is only just adequate to deal with usual demand. While this model may work for a car manufacturer, it leads to inefficiencies in a health service even without a pandemic. We are all familiar with the routine of 'winter pressures', and the often desperate efforts to identify empty (or to clear) hospital beds. If we struggle to cope in normal times, what hope is there of responding well to a pandemic? It is possible that this lack of spare resources may have been fatal to some; there are likely to be many reasons why Germany's mortality per million population is so much lower than ours, but one set of factors is that they have many more hospital beds, intensive care beds and doctors per 100 population than we do..$^{19}$ They did not therefore have to reorganise hospitals and stop non-COVID-19 procedures to the same extent that we did, nor was their nosocomial infection rate as high. Making policies which ensure we have some 'crisis resilience' would prepare us not only for medical crises, but may also improve the sustainability of the workforce in other ways - by improving training, protecting mental health, creating the ability to work across sectors, and in fostering greater understanding of competing needs. Other emergency sectors work like this: most fire engines are not used for most of the time, and certainly (thankfully) our military are not fighting most of the time.

\section{Conclusion}

We believe that the recommendations given above are necessary to guide our approach to the next stage in the pandemic. They set some practical ground norms through which we can work out the many, more specific issues that will arise - for example, how to prioritise recipients for a vaccine, how to ensure protection of staff with PPE while reducing plastic consumption, ${ }^{20}$ and how to manage visiting in hospitals and care homes. Ensuring advocacy (including for those with less powerful voices) for all of those affected, and, as a group, considering each of the principles listed ${ }^{5}$ will help to ensure decisions are equitable, transparent, and sustainable.

We welcome public debate on what additional conditions might be needed, and how we can reach consensus on non-political principles to ensure that healthcare and disease prevention are safely delivered to all those that need them throughout the next stages of the pandemic. Once agreed, these principles will help us respond logically and ethically to the next challenges we face.

\section{References}

1 Huxtable R. Bin it or pin it? Which professional ethical guidance on managing COVID-19 should I follow? BMC Med Ethics 2020;21:60

2 General Medical Council. Duties of a doctor. www.gmc-uk.org/ ethical-guidance/ethical-guidance-for-doctors/good-medicalpractice/duties-of-a-doctor [Accessed 20 September 2020].

3 Fritz Z, Huxtable R, Ives ] et al. Ethical road map through the covid-19 pandemic. BMJ 2020;369:m2033.
4 McDougall RJ, Gillam L, Ko D et al. Balancing health worker wellbeing and duty to care: an ethical approach to staff safety in COVID-19 and beyond. J Med Ethics 2020, in press (doi: 10.1136/ medethics-2020-106557).

5 Fritz Z, Cox CL. Integrating philosophy, policy and practice to create a just and fair health service J Med Ethics 2020;46:797-802.

6 Aldridge RW, Lewer D, Katikireddi SV et al. Black, Asian and Minority Ethnic groups in England are at increased risk of death from COVID-19: indirect standardisation of NHS mortality data. Wellcome Open Res 2020;5:88.

7 Li J, Huang DQ, Zou B et al. Epidemiology of COVID-19: A systematic review and meta-analysis of clinical characteristics, risk factors, and outcomes. J Med Virol 2020, in press (doi: 10.1002/jmv.26424).

8 Liddell K, Skopek JM, Palmer S et al. Who gets the ventilator? Important legal rights in a pandemic. J Med Ethics 2020;46:421-6.

9 National Institute of Health and Care Excellence. COVID-19 rapid guideline: critical care in adults. NICE guideline [NG159]. NICE, 2020. Available from www.nice.org.uk/guidance/ng159 [Accessed 30 September 2020].

10 Huppert J, Rogers de Waal J. Save keyworkers over royal family or cabinet, say public. YouGov, 2020. https://yougov.co.uk/topics/ health/articles-reports/2020/06/05/save-key-workers-over-royalfamily-or-cabinet-say- [Accessed 30 September 2020].

11 Awad E, Dsouza S, Kim R et al. The Moral Machine experiment. Nature 2018;563:59-64.

12 Esobar O, Elstub S. Forms of mini-publics: An introduction to deliberative innovations in democratic practice. New Democracy, 2017. Available from www.newdemocracy.com.au/2017/05/08/ forms-of-mini-publics/.

13 Fishkin J. When the people speak: deliberative democracy and public consultation. Oxford: Oxford University Press, 2009.

14 Berkman ND, Sheridan SL, Donahue KE et al. Low health literacy and health outcomes: an updated systematic review. Ann Intern Med 2011;155:97-107.

15 Shrank WH, Rogstad TL, Parekh N. Waste in the US health care system: estimated costs and potential for savings. JAMA 2019:322:1501-9.

16 NHS England. Interim clinical commissioning policy: grommets (children and adults). NHSE, 2013. www.england.nhs.uk/ commissioning/wp-content/uploads/sites/12/2013/11/N-SC015.pdf [Accessed 30 November 2020].

17 Rawls J. A Theory of Justice. Harvard, MA: Harvard University Press, 1971.

18 Lamont J, Favor C. Distributive justice. In: Zalta EN (ed), The Stanford Encyclopedia of Philosophy, Winter 2017 Edition. https:// plato.stanford.edu/archives/win2017/entries/justice-distributive/ [Accessed 30 November 2020].

19 Chazin G. Oversupply of hospital beds helps Germany to fight virus. Financial Times, 13 April 2020.

20 Wardrope A. Health justice in the Anthropocene: medical ethics and the Land Ethic. J Med Ethics 2020;46:791-6.

\footnotetext{
Address for correspondence: Dr Zoë Fritz, The Healthcare Improvement Studies (THIS) Institute, University of Cambridge, Clifford Allbutt Building Cambridge Biomedical Campus, Cambridge CB2 OAH, UK.

Email: Zbmf2@cam.ac.uk
} 